\title{
ON THE COMPARATIVE STUDY OF PREDICTION ACCURACY FOR CREDIT CARD FRAUD DETECTION WITH IMBALANCED CLASSIFICATIONS
}

\author{
Tahani Baabdullah, Amani Alzahrani and Danda B. Rawat
}

Data Science and Cybersecurity Center (DSC $\left.{ }^{2}\right)$

Department of Electrical Engineering and Computer Science

Howard University, Washington D.C. 20059. USA

\{Tahani.baabdullah, Amani.alzahrani, Danda.rawat\}@howard.edu

\begin{abstract}
Credit card fraud is one of the critical issues due to its significant losses to both financial institutions and individuals in the society. The accurate detection and prevention of fraudulent activities are necessary to protect financial institutions and individuals. This paper performs a comparative experimental study to detect credit card frauds, as well as to tackle the imbalance classification problem by applying different machine learning algorithms for handling imbalanced datasets. Our study shows that there is no need to process imbalance dataset by applying resampling techniques to measure the performance of our classifiers and it is sufficient to measure the performance through the three-performance measurements (Accuracy, Sensitivity, and Area Under Precision/Recall Curve (PRC)) to prove the accuracy of the prediction of classification. Finally, by handling imbalanced classifications with imbalance datasets, one can minimize the number of false alarms, reduce damages to financial institutions and individuals, increase accurate for fraud detection, and decrease the occurrence of fraud cases using machine learning techniques. Keywords: Fraud detection, fraud cybercrimes, imbalanced classification, credit card fraud, imbalanced datasets.
\end{abstract}

\section{INTRODUCTION}

Many financial institutions, such as credit card company and banks, consider fraud detection as a big issue. Recent study shows that credit card fraud costs around \$67 billion per year. Currently, there are many kinds of frauds such as insurance fraud, credit card fraud, statement fraud, securities fraud etc. The most common kind of them is credit card fraud. The definition of credit card fraud is the unauthorized use of a credit card account. Thus, the fraud occurs when the third party starts unauthorized using of the credit card without the consent of the card owner (Makki et al. 2019). The fraudsters can get goods without paying their own money, or they can access other accounts to get funds. The typical classifications of credit card fraud are listed in Table 1 (Makki et al. 2019).

Table 1: Classifications of credit card fraud.

\begin{tabular}{|l|l|}
\hline Method & Action \\
\hline Simple theft (offline fraud) & Fast and straightforward type via stealing credit card. \\
\hline Application fraud & $\begin{array}{l}\text { Using false personal information by individuals in case of have new } \\
\text { credit cards. }\end{array}$ \\
\hline Bankruptcy fraud & $\begin{array}{l}\text { Using a credit card to purchase goods even though knowing the } \\
\text { disability to pay for it. }\end{array}$ \\
\hline Internal fraud & Stealing card's information to be used by bank employees. \\
\hline
\end{tabular}


Baabdullah, Alzahrani, and Rawat

\begin{tabular}{|l|l} 
Counterfeit fraud/behavioral & Have card's information by skimming or shoulder surfing.
\end{tabular}

fraud/cardholder-not-present fraud

\subsection{Problem Description}

In general, number of fraud usage of credit card is smaller than that of legitimate usage resulting in imbalance data and classification. Imbalanced classification happens when the number of observations in one class (minority) is very small compared to the number of observations in the other class (majority) in the datasets in case of binary classification. Thus, the ratio between the two classes, fraud transactions class and legitimate/original transactions class, is very small which impacts to get inaccurate results when machine learning classification algorithms are used to detect fraud transactions. Hence, the imbalanced classification problem is considered to be one of the most crucial issues. The problem occurs because of the existence of an imbalanced and highly skewed distribution of the data between normal and fraud transactions. Generally, the imbalanced classification problem still appears since the ratio of fraudulent/criminal transactions is smaller than the normal/legitimate transactions (Makki et al. 2019), (Rawat \& Ghafoor, 2018). In our experiment, the dataset is highly imbalanced, the positive class (frauds) account is $0.17 \%$ of all transactions. Figure 1 and Figure 2 show the transaction class distribution as a class 0 for a normal transaction and class 1 for fraud transactions. It illustrates an imbalanced classification of credit cards transactions datasets according to the features: Time and Amount.

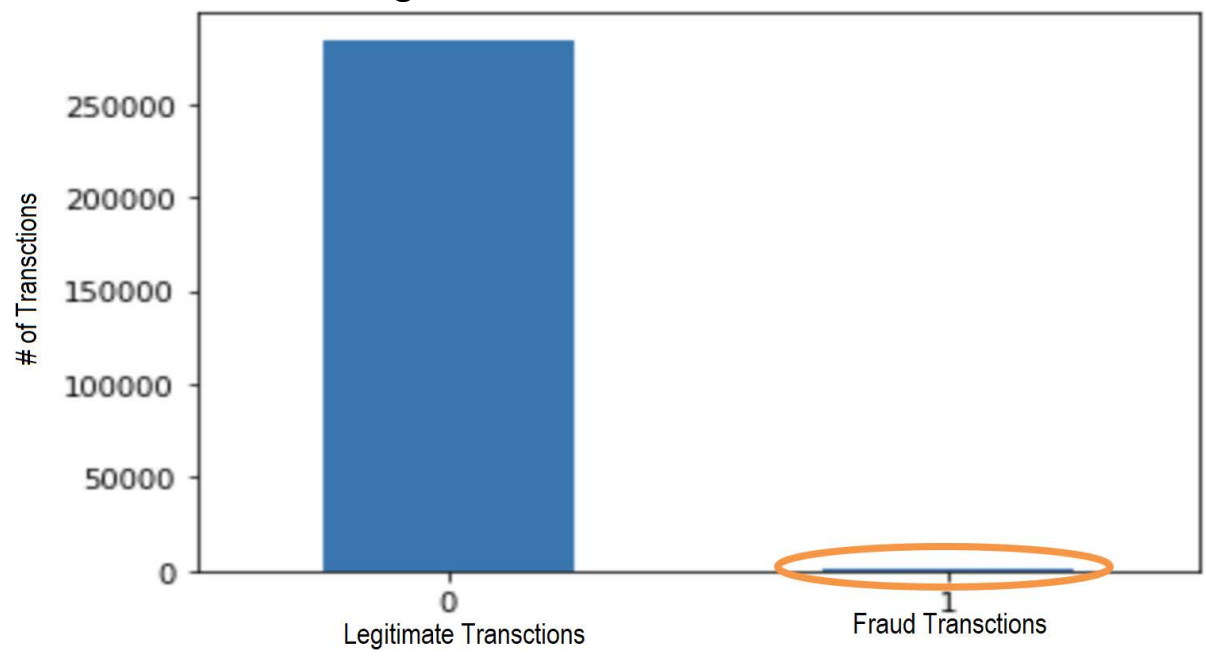

Figure 1. Transaction class distribution (class 0: Normal transaction, class 1: Fraud transaction). 


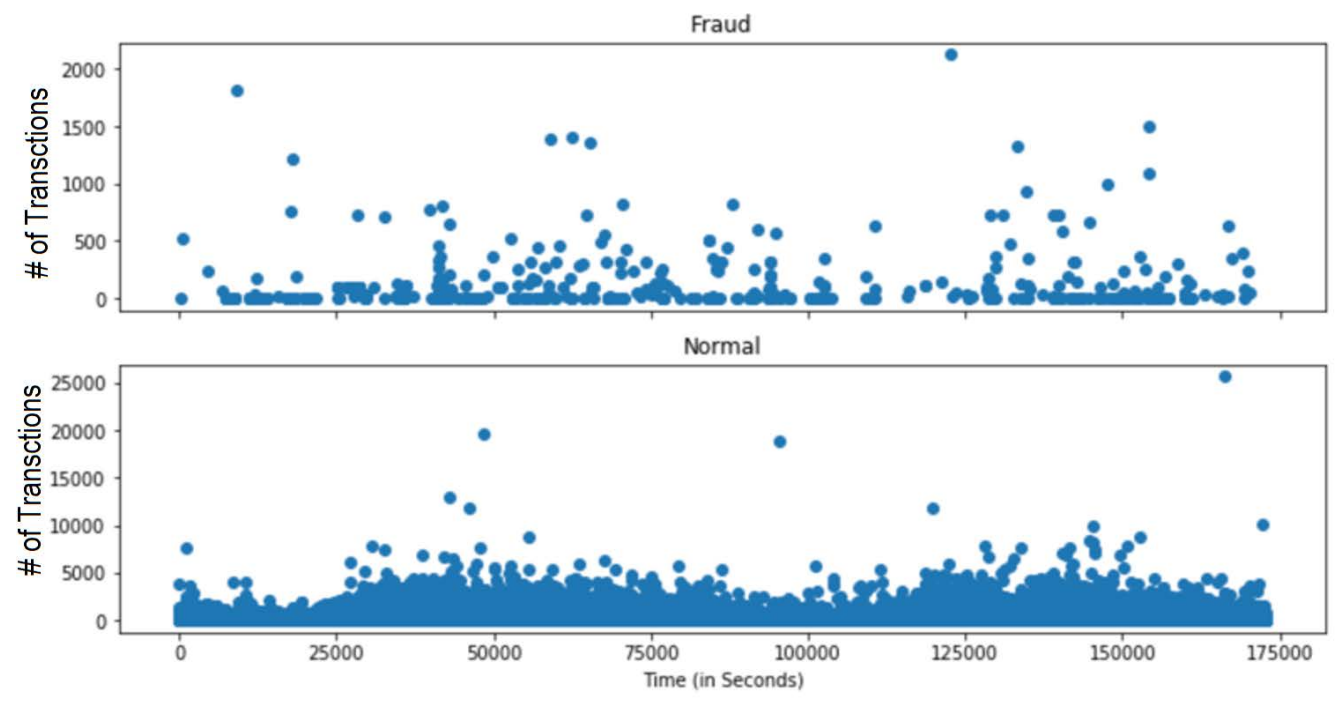

Figure 2. Amounts by seconds of Fraud and Normal Transactions.

As known, sensitivity and specificity are used as performance measures to evaluate binary classifiers, and Receiver Operating Characteristics (ROC) curves also show the performance of binary classifiers. There are other measurements of performance, such as positive predictive value (PPV) and the associated Precision/Recall Curve (PRC), but they are not used frequently as ROC curve. The ROC curves and PRC are considered diagnostic tools applied for the interpretation of probabilistic forecasts for binary classifier predictive modeling problems (Makki et al. 2019) (Saito and Marc 2015). Although the ROC curve is the most common tool used to compare models and to predict probabilities for two-class problems, it is an appropriate tool in case of balanced datasets while the precision-recall curve is suitable in case of imbalanced datasets. When datasets are imbalanced data frame between positive and negative samples, then this imbalance effects can be seen in PRC curve but it does not affect the ROC curve. The PRC curve is used with imbalanced datasets when positive and negative samples are extremely dissimilar. Therefore, the ROC curve does not measure the performance of the binary classifier model accurately. On the other hand, the PRC curve can reflect the performance of the binary classifier model precisely because it is based on the evaluation of the fraction of true positives among positive predictions (Makki et al. 2019) (Saito and Marc 2015). In this paper, an experimental study is conducted to detect the credit card fraud transactions, as well as tackle the imbalanced classification problem through applying different approaches and techniques to handle imbalanced datasets, for example, data-level approach resampling techniques. Then, the performance of the binary classifier model was measured using different measurements such as Accuracy, Sensitivity, and Area Under PRC. Then, we compared the results between numbers of classifier models.

\subsection{Paper Organization}

Rest of the paper is organized as follows. Section 2 discusses related work. Section 3 presents the system model. In section 4, the proposed approach is explained. In Section 5, experimental results are discussed. Section 6 concludes the paper.

\section{RELATED WORK}

This section explains the state of the art work related to credit card fraud detection and the imbalanced dataset problem in credit card fraud detection. 


\subsection{Credit Card Fraud Detection}

Esakkiraj and Chidambaram conducted a Hidden Markov Model (HMM) to detect fraud transactions among a sequence of credit card transactions (Esakkiraj, 2013). Another paper by Mishra et al. made some studies using HMM for the same objective (Mishra, 2013). A costsensitive decision tree method to detect credit card fraud transactions was proposed by Sahin et al. (Sahin, Bulkan, and Duman 2013). A cost-sensitive method related to Bayes minimum risk for detecting credit card fraud transactions was introduced by Bahnsen et al. (Bahnsen et al. 2013). Subashini and Chitra proposed that the model using the Decision Trees (CART) algorithm had better performance than the rest of the models used Decision Trees, Support Vector Machines with Polynomial Kernels, Logistic Regression (LR), and Bayesian Belief Networks (B.Subashini and Chitra 2013). Mahmoudi and Duman used simple linear discriminant analysis to detect fraud of credit card for the first time, then modified it to be more sensitive to false negatives by proposing a modified Fisher Discriminant Function (Mahmoudi and Duman 2015). Hormozi et al. introduced a fraud detection credit card system that executes the negative selection algorithm through using Hadoop and MapReduce paradigm (Hormozi et al. 2013). Carcillo et al introduced a method to label credit card fraud transactions by active learning strategies, and they investigated these strategies through their accuracies of performance and detection (Carcillo et al. 2018).

\subsection{Imbalanced Datasets of Credit Card Fraud Detection}

There are many issues related to credit card fraud detection. One of them is imbalanced dataset problem which impacts the prediction of the classes using machine learning. Class imbalance happens when the majority of observations are excessively higher than minority observations, then the classifier will have difficulties to detect the minority of observations appropriately. There are many approaches proposed to study the imbalance dataset and classification problem. As mentioned by Krawczyk, there are different ways to tackle skewed classifications with imbalanced datasets, such as Data-Level and Algorithm-Level approaches. The data-level approach aims to balance classes before applying any classification algorithm, such as oversampling or undersampling. While Algorithm-Level approach provides the potential to adapt existing classifier learning algorithms to strengthen learning for minority class, such as Cost-Sensitive methods (Krawczyk, 2016). One-class classification approach for solving imbalanced class which is a hybrid system of Particle Swarm Optimization and Auto-Associative Neural Network (PSOAANN) introduced by Kamaruddin and Ravi, and it was implemented in a Spark computational framework (Kamaruddin and Ravi 2016). An online fraud detection system was proposed by Wei et al.nand they confirmed the efficiency of that system with highly imbalanced datasets. Contrast pattern mining, neural network, and decision forest are the main three algorithms were included in their system, then they combined the results at the end (Wei Wei, 2012).

\section{SYSTEM MODEL}

The proposed system model includes five stages to accurately classify credit card transactions either to class 0 for original/legitimate transactions or to class 1 for fraud/illegitimate transactions. The first stage is importing the data obtained from the dataset that has been collected for big data mining and fraud detection research in University Libre de Bruxelles (ULB) (Kaggle Dataset, 2018). It contains 492 fraud out of 284,807 transactions. The second stage is preparing the data. The dataset contains 31 features, 28 of the features have been transformed with PCA. 'Time' and 'Amount' features have not been transformed. As a result, these two columns should be scaled as the other columns. 'Class' is the label that has the value 0 for normal transactions and 1 for fraud 
transactions as in (Kaggle Dataset, 2018). The third stage is splitting the dataset into training and testing sets. Then, resampling the training set using two Data-Level Approach Resampling Techniques: Synthetic Minority Oversampling technique (SMOTE) and NearMiss Under sampling Algorithms. SMOTE algorithm creates new synthetic points of the minor class to have balance classes, and NearMiss algorithm reduces the majority class to be equal to the minority class. The fourth stage is applying different classification algorithms on balance and imbalanced training set to train the model to be able to determine the class of the transaction either 0 or 1 . Finally, the last stage is evaluating each model using different approaches and measurements, such as confusion matrix, accuracy, ROC curve, and Precision-Recall curve (PRC). As shown in Figure 3 , the system model flow diagram has five stages which are in a specific order as follows: importing datasets, preparing features, using oversampling or under-sampling algorithms, training data, finally, evaluating classifier models and measuring performance.

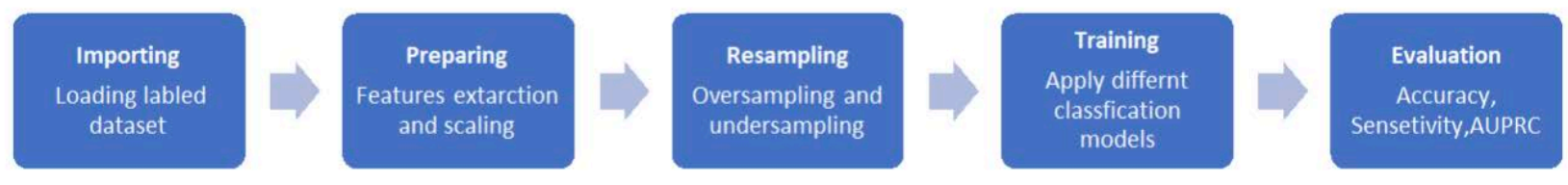

Figure 3. System Model Flow Diagram.

\section{PROPOSED APPROACH}

In this section, different techniques are applied to tackle the imbalanced classification problem. Then, each technique is evaluated to measure how it helps to improve the classification performance. Five machine learning algorithms on the baseline dataset, and on the resampled dataset (whether oversampled or under-sampled dataset) are applied. Then, the experimental results are compared and evaluated by using different measures to avoid any bias or overfitting in the model.

As known, accuracy is the most popular measure for the classification problem. However, in the credit card fraud detection transactions, usually, there are few instances of fraud compared to the legitimate credid card transactions. As a result, we can reach high accuracy by predicting the negative class every time, and ignoring the positive class which is the intended case. It is necessary to apply other metrics besides accuracy to prove the accuracy of the prediction of classification, such as sensitivity (recall), precision, and AUROC (Soni, 2018). In this experiment, the most efficient performance measures were used after analyzing results for each method to prove the accuracy of the prediction of classification.

\section{EXPERIMENT RESULTS AND PERFORMANCE EVALUATION}

In this experiment, five classifiers models are applied: Logistic Regression (LR), Gaussian Naive Bayes (NB), K-Nearest Neighbors (KNN), Decision Tree (DT), Random Forest (RF) to detect fraud transactions. Evaluating the performance of these five classifiers models is performed by comparing three performance evaluation metrics which are Sensitivity (Recall), Accuracy, and Area Under Precision-Recall Curve (AUPRC) for each classifier model in three different paradigms: baseline model, oversampling model (SMOTE), and under-sampling model (NearMiss). To get the exact values for different metrics, we have listed results of different metrics in Table 2 through Table 6 . Tables 2 to 6 display the performance results for LR, NB, KNN, DT, 
and RF classifier models including these measures: accuracy, precision, sensitivity (recall), AUPRC, and AUROC.

As shown in the tables, the accuracy for RF in the baseline model is $99 \%$ while its sensitivity is $80 \%$ and its AUPRC is $82 \%$. Also, it is illustrated that the accuracy for KNN in the baseline model is $99 \%$ while its sensitivity is $80 \%$ and its AUPRC is $80 \%$. Finally, it gives that the accuracy for NB in the baseline model $97 \%$ while its sensitivity is $86 \%$ and its AUPRC is $92 \%$. From the above results, we found that the Random Forest (RF), K-Nearest Neighbors (KNN), and Naïve Bayes (NB) classifier models have the best performance due to their highest accuracy, sensitivity, and AUPRC.

As mentioned in the beginning that our system aims to measure the performance of the five classifiers models based on the three-performance measurement (Accuracy, Sensitivity, and AUPRC) due to the imbalanced dataset problem. Therefore, imbalanced datasets lead to class imbalance (Minority class) and keep not detecting fraud transactions as well. Regarding the results of using an oversampling technique (SMOTE), we can observe that the best classifier model with highest performance is the Random Forest (RF) classifier model because of its highest results of the accuracy and AUPRC. Thus, the accuracy of RF in the SMOTE model is $99 \%$ while the sensitivity is $85 \%$ and the AUPRC is $80 \%$.

As a result of comparison of these performance measurements of the three models (baseline of datasets, SMOTE, and NearMiss) as shown in the Tables 7 to 9, we can say that there is no need to process imbalance dataset by applying resampling techniques to measure the performance of our classifiers models, and it is sufficient to measure the performance through the threeperformance measurements (Accuracy, Sensitivity, and AUPRC) to prove the accuracy of the prediction of classification.

Table 2: Performance Results for LR Classifier Models.

\begin{tabular}{|c|c|c|c|c|c|}
\hline MODEL & Accuracy & Precision & Recall & $\begin{array}{c}\text { AUPR } \\
\text { C }\end{array}$ & $\begin{array}{c}\text { AURO } \\
\text { C }\end{array}$ \\
\hline $\begin{array}{c}\text { BASELIN } \\
\text { E }\end{array}$ & 0.99 & 0.89 & 0.7 & 0.8 & 0.85 \\
\hline SMOTE & 0.97 & 0.05 & 0.91 & 0.75 & 0.95 \\
\hline $\begin{array}{c}\text { NEARMIS } \\
\text { S }\end{array}$ & 0.58 & 0.003 & 0.93 & 0.02 & 0.76 \\
\hline
\end{tabular}

Table 3: Performance Results for NB Classifier Models.

\begin{tabular}{|c|c|c|c|c|c|}
\hline MODEL & ACCURACY & PRECISION & RECALL & AUPRC & AUROC \\
\hline BASELINE & 0.97 & 0.05 & 0.86 & 0.08 & 0.92 \\
\hline SMOTE & 0.97 & 0.05 & 0.87 & 0.08 & 0.93 \\
\hline NEARMISS & 0.76 & 0.005 & 0.9 & 0.01 & 0.84 \\
\hline
\end{tabular}

Table 4: Performance Results for KNN Classifier Models.

\begin{tabular}{|c|c|c|c|c|c|}
\hline MODEL & ACCURACY & PRECISION & RECALL & AUPRC & AUROC \\
\hline BASELINE & 0.99 & 0.93 & 0.8 & 0.8 & 0.9 \\
\hline SMOTE & 0.99 & 0.59 & 0.85 & 0.63 & 0.93 \\
\hline
\end{tabular}


Baabdullah, Alzahrani, and Rawat

\begin{tabular}{|l|l|l|l|l|l|}
\hline NEARMISS & 0.56 & 0.003 & 0.93 & 0.006 & 0.75 \\
\hline
\end{tabular}

Table 5: Performance Results for DT Classifier Models.

\begin{tabular}{|c|c|c|c|c|c|}
\hline MODEL & ACCURACY & PRECISION & RECALL & AUPRC & AUROC \\
\hline BASELINE & 0.99 & 0.72 & 0.79 & 0.57 & 0.9 \\
\hline SMOTE & $\mathbf{0 . 9 9}$ & $\mathbf{0 . 3 6}$ & $\mathbf{0 . 8 2}$ & $\mathbf{0 . 3}$ & $\mathbf{0 . 9 1}$ \\
\hline NEARMISS & 0.21 & 0.001 & 0.96 & 0.001 & 0.59 \\
\hline
\end{tabular}

Table 6: Performance Results for RF Classifier Models.

\begin{tabular}{|c|c|c|c|c|c|}
\hline MODEL & ACCURACY & PRECISION & RECALL & AUPRC & AUROC \\
\hline BASELINE & 0.99 & 0.92 & 0.83 & 0.85 & 0.92 \\
\hline SMOTE & 0.99 & 0.89 & 0.85 & 0.83 & 0.93 \\
\hline NEARMISS & 0.04 & 0.001 & 0.98 & 0.57 & 0.51 \\
\hline
\end{tabular}

Table 7: Baseline Models Performance.

\begin{tabular}{|c|c|c|c|c|c|}
\hline MODEL & ACCURACY & PRECISION & RECALL & AUPRC & AUROC \\
\hline NB & 97 & 5 & 86 & 92 & 85 \\
\hline DT & 99 & 70 & 79 & 56 & 90 \\
\hline RF & 99 & 94 & 80 & 82 & 90 \\
\hline KNN & 99 & 93 & 80 & 80 & 90 \\
\hline LR & 99 & 89 & 70 & 80 & 85 \\
\hline
\end{tabular}

Table 8: SOMTE Models Performance.

\begin{tabular}{|c|c|c|c|c|c|}
\hline MODEL & ACCURACY & PRECISION & RECALL & AUPRC & AUROC \\
\hline NB & 97 & 5 & 87 & 8 & 93 \\
\hline DT & 99 & 28 & 82 & 46 & 91 \\
\hline RF & 99 & 87 & 85 & 80 & 93 \\
\hline KNN & 99 & 59 & 85 & 63 & 93 \\
\hline LR & 97 & 5 & 91 & 75 & 85 \\
\hline
\end{tabular}

Table 9: NearMiss Models Performance.

\begin{tabular}{|c|c|c|c|c|c|}
\hline MODEL & ACCURACY & PRECISION & RECALL & AUPRC & AUROC \\
\hline NB & 76 & 0 & 90 & 1 & 84 \\
\hline DT & 21 & 2 & 96 & 0 & 58 \\
\hline RF & 4 & 0 & 98 & 1 & 53 \\
\hline KNN & 56 & 0 & 93 & 1 & 75 \\
\hline LR & 58 & 0 & 93 & 2 & 76 \\
\hline
\end{tabular}


We use three performance metrics Sensitivity (Recall), Accuracy, and AUPRC measured on five different classifier models. We evaluated the performance of the five classifiers, as shown in Figurers 4 to 6 . It is obvious that the highest performance of the PRC plots is related to the Random Forest (RF) classifier model as proved through the baseline model. From the experimental results for comparing baseline, oversampling, and under-sampling models, it is obvious that the efficiency of using the three-performance measurements (Accuracy, Sensitivity, and AUPRC) is sufficient to prove the accuracy of the prediction of classification and to detect credit card fraud transactions precisely. Therefore, it is not needed to process imbalance dataset by applying resampling techniques to measure the performance of classifiers models, and using three-performance measurements (Accuracy, Sensitivity, and AUPRC) are sufficient instead.
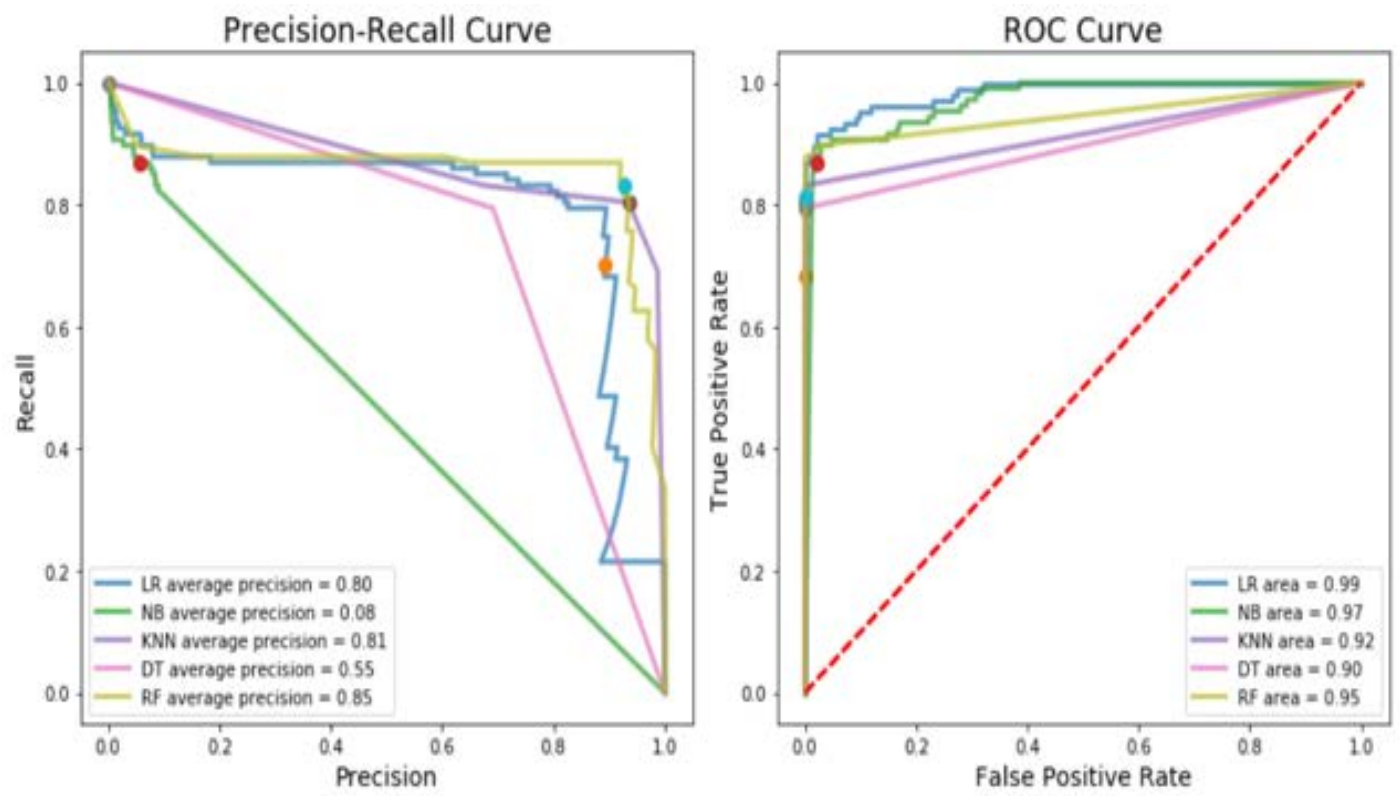

Figure 4: Baseline Performance Evaluation. 

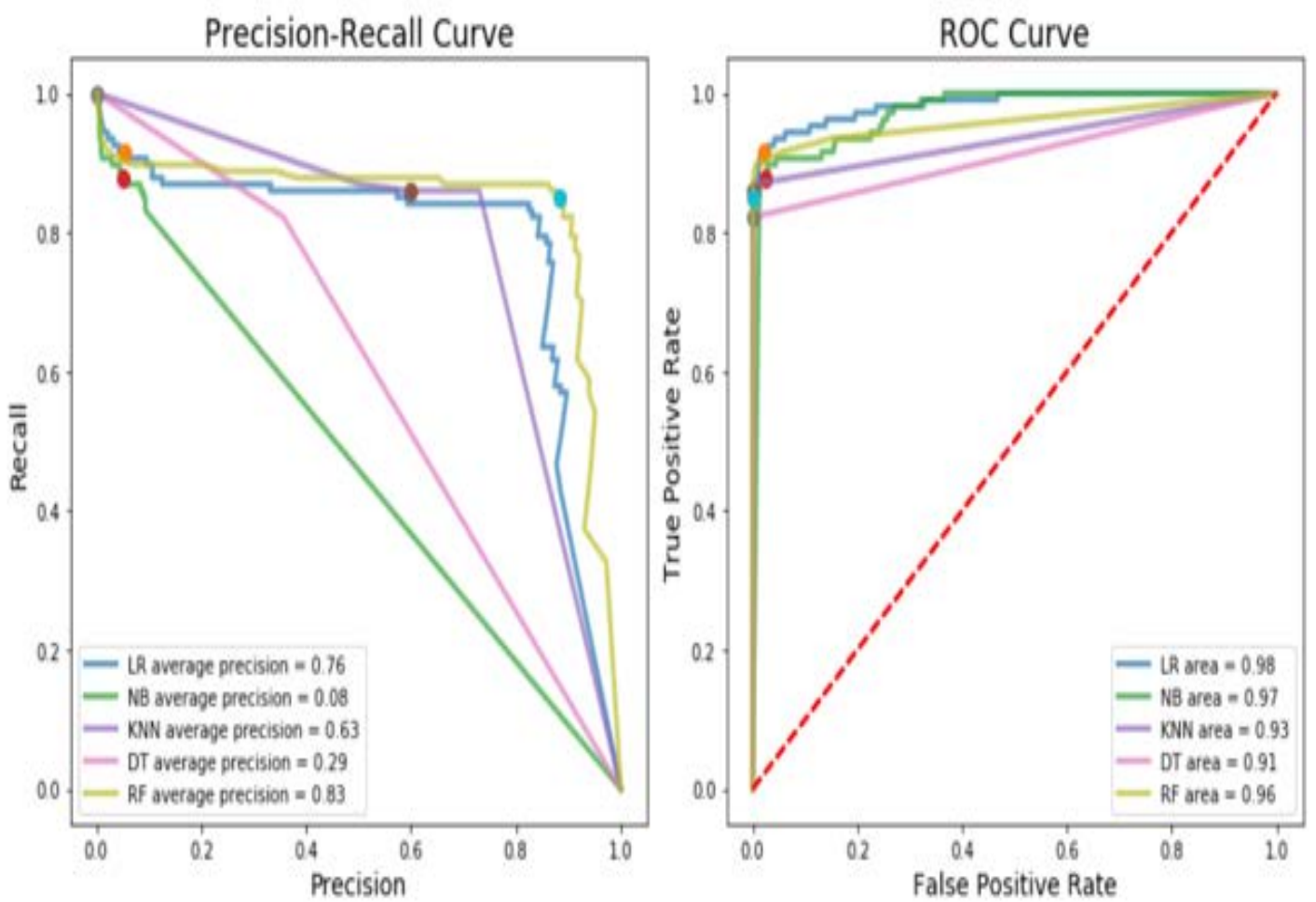

Figure 5: SMOTE Performance Evaluation.
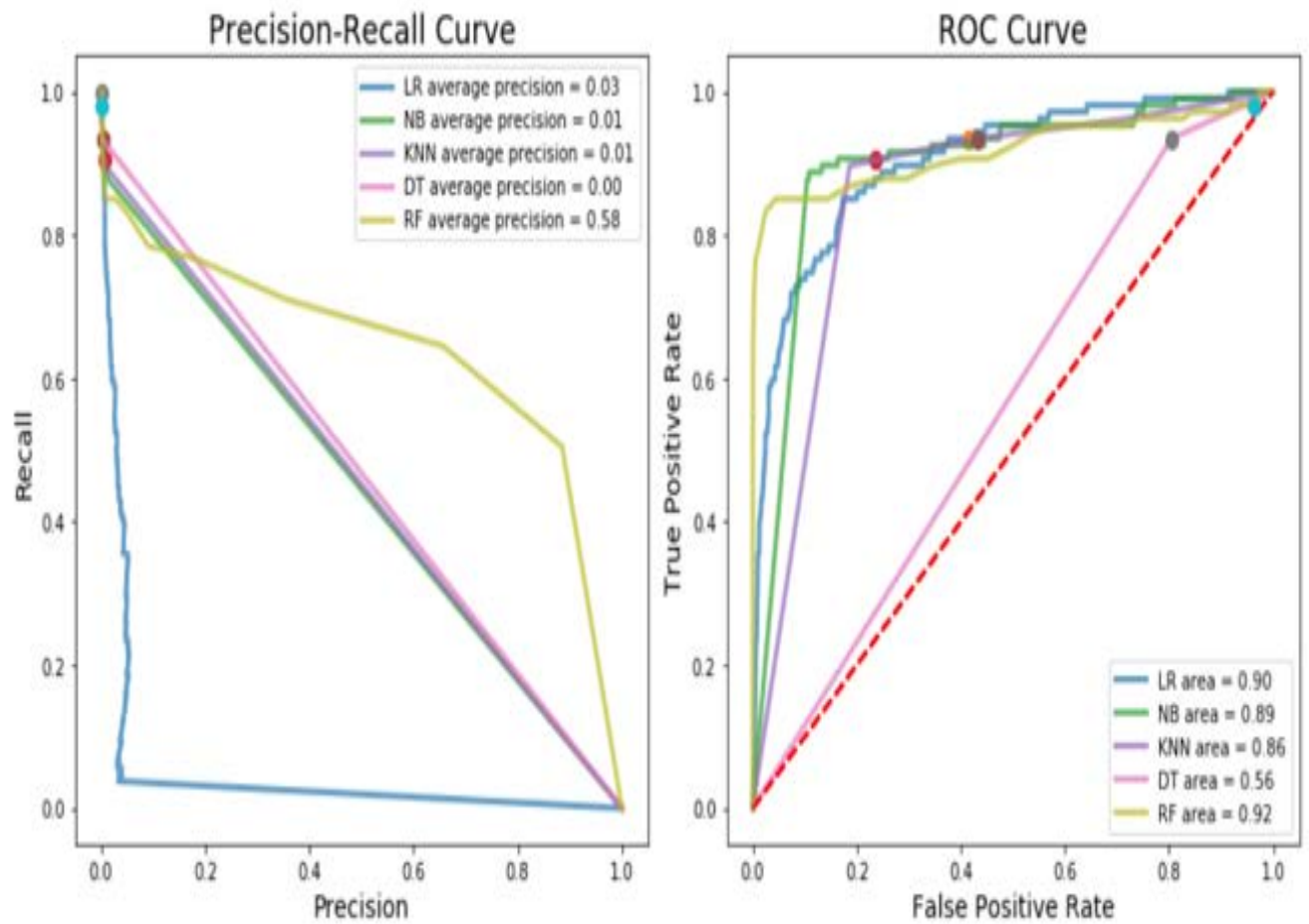

Figure 6: NearMiss Performance Evaluation. 


\section{CONCLUSION}

Credit card fraud results in huge losses to financial institutions, and businesses and individual card holders. Accurate and intensive detection and prevention of fraudulent activities are essential to protect those financial institutions and individuals with minimum false alarm and misdetection. Credit card fraud detection is challenging since there are imbalanced number of datasets for legitimate and illegitimate transactions. In this paper, we conducted an experimental study to detect the cases of credit card fraud, as well as to tackle the imbalance classification problem by applying different approaches for handling imbalanced datasets. Results suggested that there is no need to process imbalance dataset by applying resampling techniques to measure the performance of the classifiers models. It is sufficient to measure the performance through the three-performance measurements (Accuracy, Sensitivity (Recall), and AUPRC) to prove the accuracy of the prediction of classification. From the results, we found that the Random Forest (RF), K-Nearest Neighbors (KNN), and Naïve Bayes (NB) classifier models are the best methods among others due to their highest accuracy, sensitivity, and AUPRC. Finally, we can say that machine learning model can handle imbalanced classification and minimize the false alarms, reduce damages to financial institutions and individuals, increase accurate detection, and decrease the occurrence of fraud cases.

\section{ACKNOWLEDGMENTS}

The work was supported in part by the US National Security Agency (NSA) and DoE's National Nuclear Security Administration (NNSA). However, any opinion, finding, and conclusions or recommendations expressed in this document are those of the authors and should not be interpreted as necessarily representing the official policies, either expressed or implied, of the funding agencies.

\section{REFERENCES}

B. Subashini, D. K. (2013). "Enhanced System for Revealing Fraudulence in Credit Card Approval.". International Journal of Engineering Research \& Technology (IJERT).

Bahnsen, A. C. (2013). "Cost sensitive credit card fraud detection using Bayes minimum risk.". 12th international conference on machine learning and applications, (pp. vol. 1, pp. 333338). IEEE.

Carcillo, F. Y.-A. (2018). Streaming active learning strategies for real-life credit card fraud detection: assessment and visualizationI ,. nternational Journal of Data Science and Analytics 5, no. $4: 285-300$.

Esakkiraj, S. a. (2013). A predictive approach for fraud detection using hidden Mar-kov model. International Journal of Engineering Research \& Technology 2, 1-7.

Hormozi, H. M. (2013). Credit cards fraud detection by negative selection algorithm on hadoop (To reduce the training time). The 5th Conference on Information and Knowledge Technology (pp. pp. 40-43). IEEE.

Kaggle Dataset. (2018). Retrieved Nov 12, 2019, from https://www.kaggle.com/mlgulb/creditcardfraud 
Kamaruddin, S. a. (2016). Credit card fraud detection using big data analytics: use of PSOAANN based one-class classification. Proceedings of the International Conference on Informatics and Analytics, (pp. pp. 1-8.).

Krawczyk, B. (2016). Learning from imbalanced data: open challenges and future directions. Progress in Artificial Intelligence 5, (pp. no. 4: 221-232.).

Mahmoudi, N. a. (2015). Detecting credit card fraud by modified Fisher discrimi-nant analysis,. Expert Systems with Applications 42, (pp. no. $5: 2510-2516$.).

Makki, S. Z.-S. (2019). An Experimental Study With Imbalanced Classification Approaches for Credit Card Fraud Detection. IEEE.

Martinez, J. (2019, July). Credit Fraud || Dealing with Imbalanced Datasets. Retrieved 11 1, 2019, from https://www.kaggle.com/janiobachmann/credit-fraud-dealing-with-imbalanceddatasets

Mishra, J. S. (2013). "A novel approach for credit card fraud detection targeting the Indian market,". Intl Journal of Computer Science Issues, p. 172,.

Rawat, D. B., \& Ghafoor, K. Z. (2018). Smart Cities Cybersecurity and Privacy. New York: Elsevier.

Sahin, Y. S. (2013). "A cost-sensitive decision tree approach for fraud detection.". (pp. no. 15 : 5916-5923.). Expert Systems with Applications 40.

Saito, T. a. (2015). The precision-recall plot is more informative than the ROC plot when evaluating binary classifiers on imbalanced datasets. PloS one.

Soni, D. (2018, Feb 2). Dealing with imbalanced classes in machine learning. Retrieved December 10 , 2019, from towardsdatascience: https://towardsdatascience.com/dealing-withimbalanced-classes-in-machine-learning-d43d6fa19d2

Wei Wei, J. L. (2012). Effective detection of sophisticated online banking fraud on extremely imbalanced data I . Internet and Web Information Systems. Springer Science+Business Media, LLC.

\section{AUTHOR BIOGRAPHIES}

TAHANI BAABDULLAH is pursuing her $\mathrm{PhD}$ in Computer Science in the Department of Electrical Engineering and Computer Science at Howard University, Washington DC, USA, under the supervision of Dr. Danda B. Rawat. She is associated with Howard University Data Science and Cybersecurity Center (DSC2). Her research interests lie in Machine Learning, Internet of Things and Cybersecurity. Ms. Tahani Baabdullah is on her study leave from Princess Nourah Bint Abdulrahman University (PNU). Her email is tahani.baabdullah@bison.howard.edu.

AMANI ALZAHRANI is pursuing her $\mathrm{PhD}$ in Computer Science in the Department of Electrical Engineering and Computer Science at Howard University, Washington DC, USA, under the supervision of Dr. Danda B. Rawat. She is associated with Howard University Data Science and Cybersecurity Center (DSC2). Her research interests lie in Machine Learning and Cybersecurity. 
Ms. Amani Alzahrani is on her study leave from Shaqra University. Her email is amani.alzahrani@bison.howard.edu.

DANDA B. RAWAT is an Associate Professor in the Department of Electrical Engineering and Computer Science at Howard University. Dr. Rawat is the Founding Director of the Howard University Data Science and Cybersecurity Center (DSC2), Director of Cybersecurity and Wireless Networking Innovations (CWiNs) Research Lab, Graduate Program Director of Howard-CS Graduate Programs and Director of Graduate Cybersecurity Certificate Program at Howard University, Washington, DC, USA. Dr. Rawat is engaged in research and teaching in the areas of cybersecurity, machine learning and wireless networking for emerging networked systems including cyber-physical systems, Internet-of-Things, smart cities, software defined systems and vehicular networks. He has secured over \$4 million in research funding from US National Science Foundation, US Department of Homeland Security, Department of Energy, National Nuclear Security Administration (NNSA), DoD Research Labs, Industry (Microsoft, Intel, etc.) and private Foundations. Dr. Rawat is the recipient of NSF CAREER Award in 2016, the US Air Force Research Laboratory (AFRL) Summer Faculty Visiting Fellowship in 2017, Outstanding Research Faculty Award (Award for Excellence in Scholarly Activity) at GSU in 2015, the Best Paper Awards and Outstanding PhD Researcher Award in 2009. He has delivered over 15 Keynotes and invited speeches at international conferences and workshops. Dr. Rawat received the Ph.D. degree from Old Dominion University, Norfolk, Virginia. Dr. Rawat is a Senior Member of IEEE and ACM, a member of ASEE and AAAS, and a Fellow of the Institution of Engineering and Technology (IET). His email is Danda.Rawat@howard.edu. 